\title{
A THEOREM ON CONTINUED FRACTIONS
}

\section{IRVING REINER}

1. Introduction. Let $\left[a_{1}, \cdots, a_{n}\right]$ denote the simple continued fraction whose successive quotients $a_{1}, \cdots, a_{n}$ are elements of a field $K$. Following the procedure of Milne-Thompson, ${ }^{1}$ define the formal numerator $P$ and formal denominator $Q$ of this continued fraction by means of

$$
\left(\begin{array}{ll}
a_{1} & 1 \\
1 & 0
\end{array}\right)\left(\begin{array}{ll}
a_{2} & 1 \\
1 & 0
\end{array}\right) \cdots\left(\begin{array}{ll}
a_{n} & 1 \\
1 & 0
\end{array}\right)\left(\begin{array}{l}
1 \\
0
\end{array}\right)=\left(\begin{array}{l}
P \\
Q
\end{array}\right) .
$$

For brevity, we write $\left[a_{1}, \cdots, a_{n}\right] \sim P / Q$.

Now let $K$ be a skew-field, and let $R=K[x]$ be the ring of polynomials in an indeterminate $x$ with coefficients in $K$, where we assume that $x$ commutes with all elements of $K$. For $f_{1}, \cdots, f_{n} \in R$, define $\left[f_{1}, \cdots, f_{n}\right] \sim P / Q$ where (as above)

$$
\left(\begin{array}{cc}
f_{1} & 1 \\
1 & 0
\end{array}\right)\left(\begin{array}{cc}
f_{2} & 1 \\
1 & 0
\end{array}\right) \cdots\left(\begin{array}{cc}
f_{n} & 1 \\
1 & 0
\end{array}\right)\left(\begin{array}{l}
1 \\
0
\end{array}\right)=\left(\begin{array}{l}
P \\
Q
\end{array}\right) .
$$

Next let $f \rightarrow f^{*}$ denote any homomorphism of $(R,+)$ into itself which leaves $K$ elementwise fixed, and satisfies $(a f)^{*}=a f^{*}$ for all $a \in K, f \in R$. We shall prove:

TheOREM. If $f_{1}, \cdots, f_{k} \in R$ are such that $\left[f_{1}, \cdots, f_{k}\right] \sim P / Q$ where $P, Q \in K$, then also $\left[f_{1}^{*}, \cdots, f_{k}^{*}\right] \sim P / Q$.

Thus, any identity of the form $\left[f_{1}, \cdots, f_{k}\right] \sim P / Q, P, Q \in K$, depends only upon the additive structure of $R$, and not upon its multiplicative structure. This result will be basic in a future paper on the automorphisms of $G L_{2}(R) .^{2}$

2. Several lemmas will be needed for the proof of the theorem.

Lemma 1. Let $\left[f_{1}, \cdots, f_{n}\right] \sim P / Q$, where $f_{1}, \cdots, f_{n} \in R$. Suppose that $f_{r}, f_{r+1}, \cdots, f_{s}(s \geqq r)$ satisfy

$$
f_{r}, \cdots, f_{s} \in K, \quad\left(\begin{array}{cc}
f_{r} & 1 \\
1 & 0
\end{array}\right) \cdots\left(\begin{array}{cc}
f_{s} & 1 \\
1 & 0
\end{array}\right)=\left(\begin{array}{ll}
0 & c \\
b & d
\end{array}\right) .
$$

Set $e=d c^{-1}, f_{s+1}^{\prime}=b f_{s+1} c^{-1}, f_{s+2}^{\prime}=c f_{s+2} b^{-1}, \ldots$ Then

Presented to the Society April 6, 1957; received by the editors March 18, 1957.

1 Proceedings of the Edinburgh Mathematical Society, 1933.

${ }^{2}$ I. Reiner, $A$ new type of automorphism of the general linear group over a ring, to appear in Annals of Mathematics. 


$$
\begin{gathered}
\left(\begin{array}{cc}
f_{1} & 1 \\
1 & 0
\end{array}\right) \cdots\left(\begin{array}{cc}
f_{r-2} & 1 \\
1 & 0
\end{array}\right)\left(\begin{array}{cc}
f_{r-1}+e+f_{s+1}^{\prime} & 1 \\
1 & 0
\end{array}\right)\left(\begin{array}{cc}
f_{s+2}^{\prime} & 1 \\
1 & 0
\end{array}\right) \cdots \\
\left(\begin{array}{cc}
f_{n}^{\prime} & 1 \\
1 & 0
\end{array}\right)\left(\begin{array}{l}
t \\
0
\end{array}\right)=\left(\begin{array}{l}
P \\
Q
\end{array}\right),
\end{gathered}
$$

where $t$ is either $b$ or $c$, depending on the parity of $n-s$.

Proof. From (2) we have

$$
\begin{aligned}
& \left(\begin{array}{l}
P \\
Q
\end{array}\right) \\
& =\left(\begin{array}{ll}
f_{1} & 1 \\
1 & 0
\end{array}\right) \cdots\left(\begin{array}{cc}
f_{r-1} & 1 \\
1 & 0
\end{array}\right)\left(\begin{array}{ll}
0 & 1 \\
1 & e
\end{array}\right)\left(\begin{array}{ll}
b & 0 \\
0 & c
\end{array}\right)\left(\begin{array}{cc}
f_{s+1} & 1 \\
1 & 0
\end{array}\right) \cdots\left(\begin{array}{cc}
f_{n} & 1 \\
1 & 0
\end{array}\right)\left(\begin{array}{l}
1 \\
0
\end{array}\right) \\
& =\left(\begin{array}{ll}
f_{1} & 1 \\
1 & 0
\end{array}\right) \cdots\left(\begin{array}{cc}
f_{r-1} & 1 \\
1 & 0
\end{array}\right)^{\prime}\left(\begin{array}{ll}
0 & 1 \\
1 & e
\end{array}\right)\left(\begin{array}{cc}
f_{s+1}^{\prime} & 1 \\
1 & 0
\end{array}\right) \cdots\left(\begin{array}{cc}
f_{n}^{\prime} & 1 \\
1 & 0
\end{array}\right)\left(\begin{array}{l}
t \\
0
\end{array}\right),
\end{aligned}
$$

which readily implies (4).

Lemma 2. Let $f_{1}, \cdots, f_{n} \in R, f_{1} \notin K, f_{n} \notin K, \quad n>1$. Define $\left[f_{i}, \cdots, f_{n}\right] \sim P_{i} / Q_{i}, 1 \leqq i \leqq n$. If both $P_{1}$ and $Q_{1}$ lie in $K$, then there exists a run of consecutive elements $f_{r}, \cdots, f_{s}(1<r \leqq s<n)$ such that (3) holds.

Proof. Suppose that for every run of consecutive elements $f_{r}, \cdots, f_{s}$ which lie in $K$, we have $\left[f_{r}, \cdots, f_{s}\right] \sim a / b$ with $a \neq 0$. We shall obtain a contradiction. Using (2), we have

$$
P_{i}=f_{i} P_{i+1}+Q_{i+1}, \quad Q_{i}=P_{i+1}, \quad 1 \leqq i \leqq n-1 .
$$

Consequently

(5) If $\operatorname{deg} P_{i} \leqq \operatorname{deg} Q_{i}$, and if $f_{i} \notin K$, then $\operatorname{deg} P_{i+1}<\operatorname{deg} Q_{i+1}$.

Since $\operatorname{deg} P_{1} \leqq \operatorname{deg} Q_{1}$, and $f_{1} \notin K$, (5) shows that $\operatorname{deg} P_{2}<\operatorname{deg} Q_{2}$. If also $f_{2} \notin K$, we have likewise: $\operatorname{deg} P_{3}<\operatorname{deg} Q_{3}$. We may continue this process until we reach the first of the elements $f_{1}, f_{2}, \cdots$ which lies in $K$, say $f_{r}$; then $\operatorname{deg} P_{r}<\operatorname{deg} Q_{r}$. Let $s$ be chosen so that $f_{r}, \cdots$, $f_{s} \in K, f_{s+1} \notin K$. By the supposition at the beginning of the proof, we have $\left[f_{r}, \cdots, f_{s}\right] \sim a / b$ with $a \neq 0$. Setting

$$
\left(\begin{array}{ll}
f_{r} & 1 \\
1 & 0
\end{array}\right) \cdots\left(\begin{array}{ll}
f_{s} & 1 \\
1 & 0
\end{array}\right)=\left(\begin{array}{ll}
a & c \\
b & d
\end{array}\right),
$$

we have at once 


$$
P_{r}=a P_{s+1}+c Q_{s+1}, \quad Q_{r}=b P_{s+1}+d Q_{\delta+1} .
$$

Since deg $P_{r}<\operatorname{deg} Q_{r}$, surely $\operatorname{deg} P_{s+1}>\operatorname{deg} Q_{\varepsilon+1}$ is impossible. Hence $\operatorname{deg} P_{s+1} \leqq \operatorname{deg} Q_{s+1}$.

We may therefore continue this process of descent, eventually obtaining $\operatorname{deg} P_{n} \leqq \operatorname{deg} Q_{n}$. However, $P_{n}=f_{n}, Q_{n}=1$, and $f_{n} \notin K$, so we have reached a contradiction. This completes the proof of the lemma.

We now prove the theorem stated in $\S 1$, by induction on $k$. The theorem obviously holds for $k=1$. Suppose it proved for $1 \leqq k \leqq n-1$, where $n \geqq 2$, and suppose that $\left[f_{1}, \cdots, f_{n}\right] \sim P / Q$, where both $P$ and $Q$ lie in $K$; we shall show that $\left[f_{1}^{*}, \cdots, f_{n}^{*}\right] \sim P / Q$.

To begin with, assume that $f_{1} \in K$. Then (2) implies

$$
\left(\begin{array}{cc}
f_{2} & 1 \\
1 & 0
\end{array}\right) \cdots\left(\begin{array}{ll}
f_{n} & 1 \\
1 & 0
\end{array}\right)\left(\begin{array}{l}
1 \\
0
\end{array}\right)=\left(\begin{array}{rr}
0 & 1 \\
1 & -f_{1}
\end{array}\right)\left(\begin{array}{l}
P \\
Q
\end{array}\right) .
$$

By the induction hypothesis this remains valid when each $f_{i}(1 \leqq i \leqq n)$ is replaced by $f_{i}^{*}$; hence (2) also remains valid under this replacement.

Secondly, assume that $f_{n} \in K$. Since the result follows trivially from the induction hypothesis when $f_{n}=0$, we need only consider the case where $f_{n} \neq 0$. Equation (2) may then be written as

$$
\begin{aligned}
& \left(\begin{array}{c}
P \\
f_{n}^{-1}
\end{array}\right)=\left(\begin{array}{cc}
f_{1} f_{n} & 1 \\
1 & 0
\end{array}\right)\left(\begin{array}{cc}
f_{n}^{-1} f_{2} & 1 \\
1 & 0
\end{array}\right) \cdots\left(\begin{array}{cc}
f_{n}^{-1} f_{n-2} & 1 \\
1 & 0
\end{array}\right) \\
& \times\left(\begin{array}{cc}
f_{n-1} f_{n}+1 & 1 \\
1 & 0
\end{array}\right)\left(\begin{array}{l}
1 \\
0
\end{array}\right),
\end{aligned}
$$

provided that $n$ is even, with an analogous result for odd $n$. By the induction hypothesis, this remains valid under the substitution $f \rightarrow f^{*}$, which again implies the desired result.

We are thus left with the case where $n \geqq 2, f_{1} \notin K, f_{n} \notin K$, and $\left[f_{1}, \cdots, f_{n}\right] \sim P / Q$ with both $P$ and $Q$ in $K$. By Lemma 2 , there exists a run of consecutive elements $f_{r}, \cdots, f_{s}(s \geqq r)$ satisfying (3). Keeping the notation of Lemma 1, equation (2) implies the validity of (4), with $t \neq 0$. Upon multiplying the successive quotients $f_{n}^{\prime}, \cdots, f_{1}$ in (4) by $t$ or $t^{-1}$ (according to the method used in the preceding paragraph), equation (4) reduces to an equation of type (2). This new equation of type (2) remains true under the substitution $f \rightarrow f^{*}$, as a consequence of the induction hypothesis. Therefore also (4) remains true under $f \rightarrow f^{*}$; as above, we deduce that the original equation (2) remains correct under the substitution $f \rightarrow f^{*}$. This completes the proof of the theorem.

UNIVERSITY OF ILLINOIS 